\title{
The IBL Readout System
}

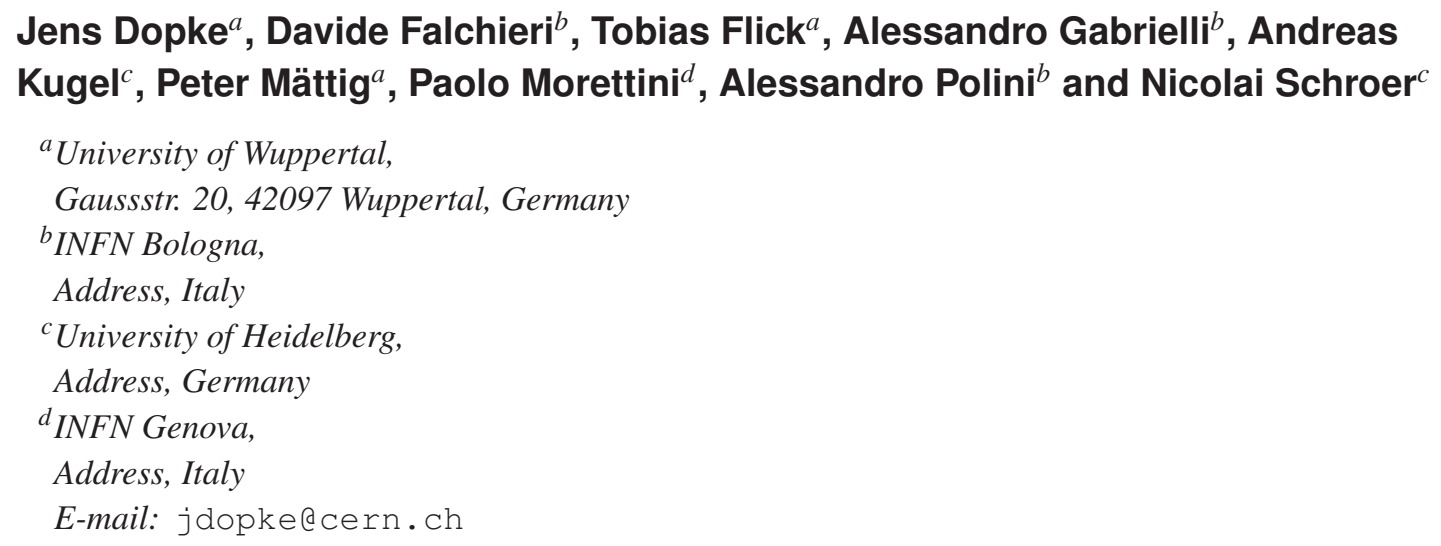

\begin{abstract}
The first upgrade for the ATLAS Pixel Detector will be an additional layer, which is called IBL (Insertable B-Layer). To readout this new layer, having new electronics, an update of the readout electronics is necessary. The aim is to develop a system which is capable to read out at a higher bandwidth, but also compatible with the existing system to be integrated into it.

This paper will describe the necessary development to reach a new readout system, concentrating on the requirements of a newly designed Back of Crate card as the optical interface in the counting room.
\end{abstract}

KEYWORDS: Digital signal processing (DSP), Optical detector readout concepts. 


\section{Contents}

1. Introduction [1]

2. IBL Readout Requirements

3. Back of Crate Card 2

3.1 Firmware Encoded Frontend Connection 3

3.2 Data Receiver 3

3.3 Embedded S-Link

3.4 Internal Interfaces

母. Testbed 5

5. Conclusions

\section{Introduction}

In the scope of the LHC phase I upgrade, increased luminosity is expected within the experiments, leading to a decrease of the ATLAS Pixel Detector [1] performance. In particular, separation of pileup events will be increasingly difficult and higher module occupancies will decrease the readout efficiency.

To overcome these deficiencies, a fourth pixelised layer, the Insertable B-Layer [2] (IBL), will be inserted into the recent Pixel Detector during the LHC phase I upgrade. Insertion is possible due to a new, smaller radius beampipe design, that will come with the IBL. The new layer will provide an increased intrinsic resolution in $z$ and increased $b$-tagging performance.

The IBL will be built in 14 staves with 16 modules each. A module unit will be built from two FrontEnd I4 (FE-I4) [3] integrated circuits, bump bonded to pixel sensors. Command and readout will be transmitted optically between the off-detector systems and the inner detector endplate. The last $6 \mathrm{~m}$ of connection are established via an electrical transmission line.

\section{IBL Readout Requirements}

Being run as part of the Pixel Detector subsystem, but based on the new FE-I4, the IBL requires multiple changes of the readout system for being operated. Each Timing, Trigger and Command (TTC) link connects to both frontends within a module. An addressing scheme is used to send configuration data or commands to individual frontends. On its data link, opposed to the previous Pixel Detector transmission system, the IBL will operate at a rate of $160 \mathrm{Mbit} / \mathrm{s}$ per frontend and encode data using the $8 \mathrm{~B} / 10 \mathrm{~B}$ scheme. 
Staying close to the Pixel Detector system, reutilising what is possible, the new readout system for IBL will be placed within a $\mathrm{VME}^{1}$ crate. A ReadOut Driver (ROD) will connect to the VME bus interface on connectors P1 and P2. The optical I/O interface will be delivered by the Back Of Crate (BOC) card connected to the ROD via connectors P2 and P3. A schematic outline of the two cards is shown in Figure 1. All critical connections within this scheme are wired up in the backside of a rack such that standard users do not interfere with them. The frontside will instead deliver status displays, probably in form of LEDs.

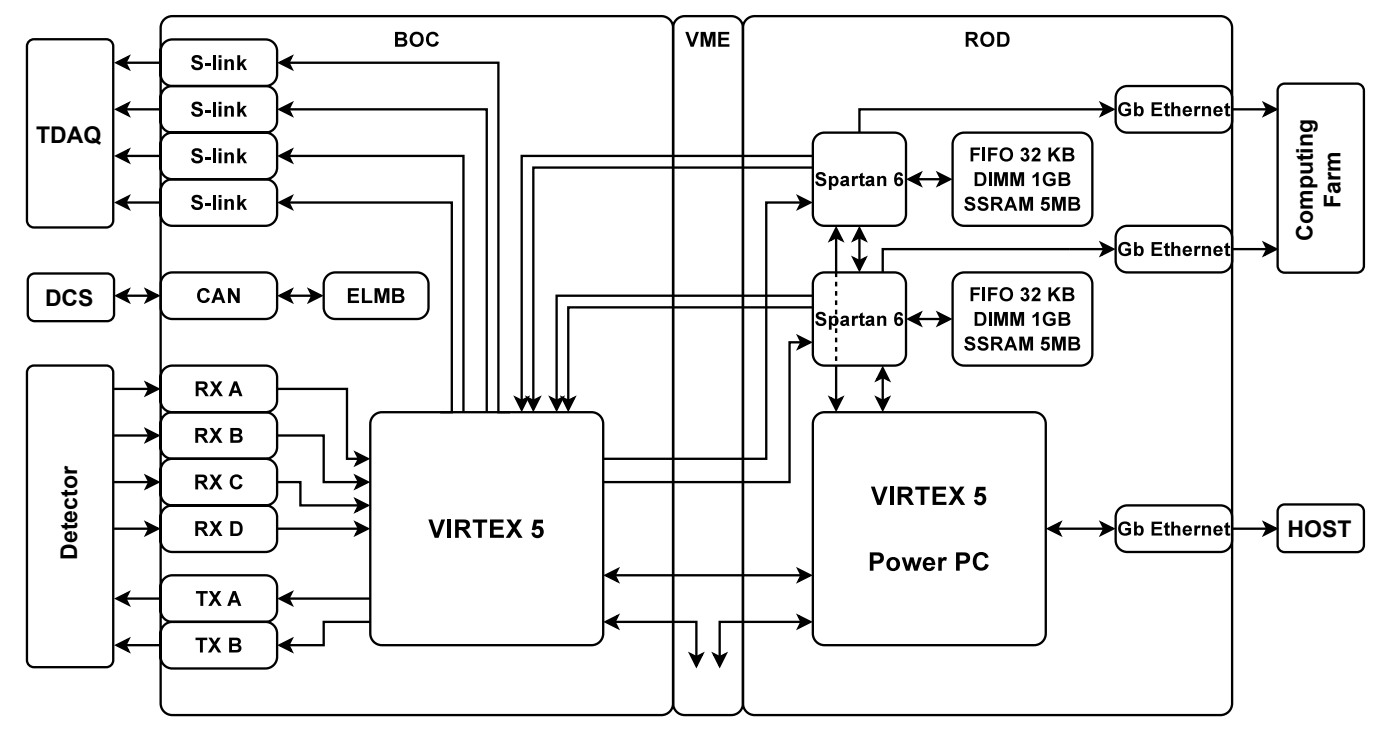

Figure 1. The ATLAS IBL readout building block [2]. The system is shown in schematic view, with all detector and readout connections to the left side (back of crate) and user-pluggable connections (Ethernet) to the right. This allows easy protection of critical connections within the crate by an interlocked door on the backside of the racks.

A total of 16 building blocks can be fitted into a VME crate. The full crate then needs an additional card to interface the TTC system and at least one ROD Crate Controller (RCC). Using the previously used ATLAS TTC Interface Module (TIM), the external crate connections stay as they are for Pixel. The following section will go into details concerning the implementation of the IBL BOC. An introduction to the IBL ROD is given in [四].

\section{Back of Crate Card}

A single BOC card serves 16 IBL modules with a total of 32 FE-I4 data links. The optical I/O will be set up in 2 transmitter and 4 receiver components, each delivering 12 channels. Multiplexing of lines will enable the system to cope with failing links online ${ }^{2}$, not needing direct exchange of components in case of failure.

\footnotetext{
${ }^{1}$ Versa Module Eurocard, standardized as ANSI/IEEE 1014-1987

${ }^{2}$ Assuming equal functionality within the on-detector transceiver component
} 


\subsection{Firmware Encoded Frontend Connection}

The IBL TTC connection will be established via a split TTC transmitter component. The encoder will be situated in firmware, while the optical transmitter will be an off the shelf component.

The firmware encoder delivers a BiPhase Mark encoded stream carrying clock and data to the detector. It allows for delaying the signal with an appropriate step size below $1 \mathrm{~ns}$ and ranges of more than one LHC clock cycle. Changing the optical mark to space ratio is available, but will probably not be necessary, because modern transmitters deliver a better translation from electrical to optical signals and the on-detector system refurbishes the received clock using a local PLL.

\subsection{Data Receiver}

As the IBL will return a balanced 8B/10B encoded stream at $160 \mathrm{Mbit} / \mathrm{s}$ per link, the new receiver circuitry can implement several advantages over the previous system:

- AC coupled receiver logic: Due to the balanced encoding, the receiver circuitry can run AC coupled. It therefore delivers a bias free stream, automatically adapting to subtle changes in the data transmission line. A TIA ${ }^{3}$ can be utilised in the first receiver stage, increasing the maximum frequency performance.

- Transition based code: Regular transitions within the received stream allow to automatically synchronise the received data with a local clock, embedding standard synchronisation techniques into the BOCs central programmable device.

- Bytewise Encoding: The receiver can be split into an $8 \mathrm{~B} / 10 \mathrm{~B}$ decoder, delivering bytewise output and a formatting section, knowing the frontend data coding scheme. Therefore the individual logic blocks will get simpler in structure and easier to debug.

For all of this, the receiver logic block will be constructed as shown in Figure 2 . Opposed to the previous BOCs usage, the IBL BOC will deliver packaged data to the ROD and take care of initial buffering.

\subsection{Embedded S-Link}

As for the detector link, the S-Link will be run from a local firmware based encoder. The optical transceiver component will not be changed, delivering the same cable connection that is served from the Pixel Detector subsystem. The total data rate of $640 \mathrm{MB} / \mathrm{s}$ will be delivered by a single $1 \mathrm{x} 4 \mathrm{SFP}^{4}$ cage and $4 \mathrm{MGTs}^{5}$ within the BOCs central programmable device. A copy of those streams will be available on a second $1 \mathrm{x} 4 \mathrm{SFP}$ cage, to deliver IBL data to an FTK ${ }^{6}$.

\footnotetext{
${ }^{3}$ TransImpedance Amplifier

${ }^{4}$ Small Form-factor Pluggable

${ }^{5}$ Multi Gigabit Transceiver

6 Fast Tracker system
} 


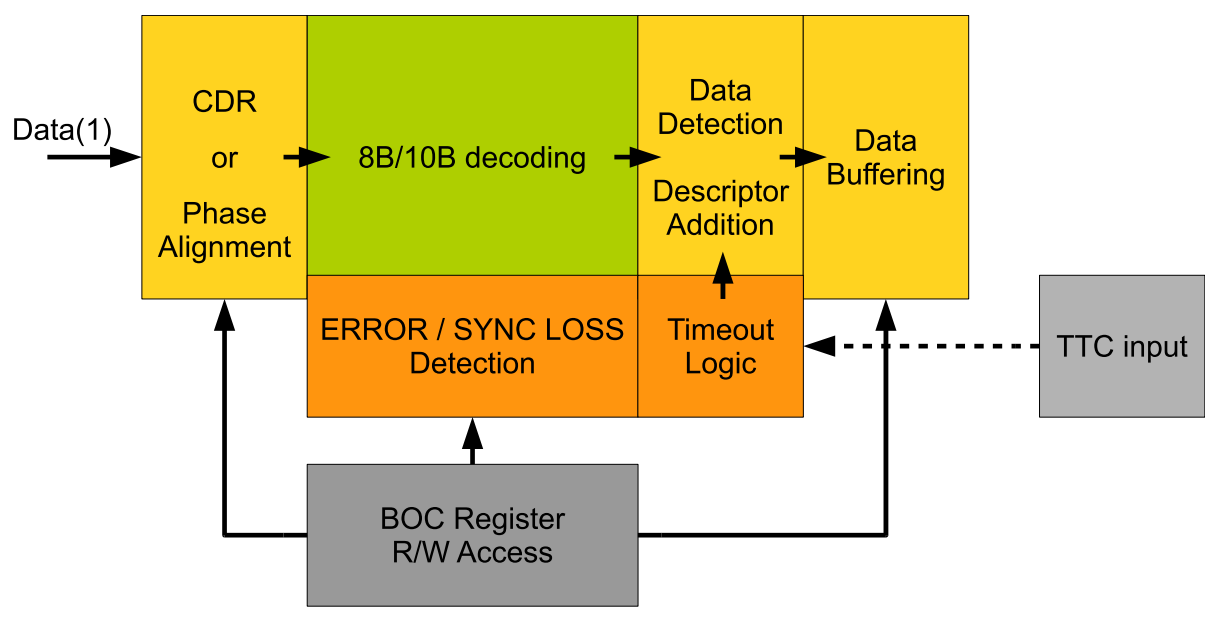

Figure 2. The IBL data receiver logic block. Input data from the detector comes in from the left, is aligned, sampled and decoded. Afterwards all IDLE statements are dropped and only data within a Start of Frame/End of Frame are handed to the output buffer block. Two blocks aside, deliver error monitoring of the stream and timeout logic to determine whether an expected packet did not arrive in time (replacing it with a timeout packet).

\subsection{Internal Interfaces}

Delivering the interfaces described above to the ROD will require an increase of bandwidth within the backplane and (to lower the maximum needed data rate) usage of the previously unused VME P0 connector. Transmissions through the backplane can be categorised into control operations, command transfer to the detector, data transfer from the detector and event transfer through the S-Link interface.

Setup Bus Control of the BOC is taken via the setup bus. This is an asynchronous bus, used in the Pixel Detector readout system. It runs with parallel 8 bit data, 10 bit address and 3 control lines. The first line controls $\mathrm{r} / \mathrm{w}$ operations, the second is the asynchronous strobe request and the third delivers an acknowledge signal to the controlling device.

TTC connection The TTC connection is directly routed into the BiPhase Mark encoder. It will decrease in number of used transfer lines from 48 to 16.

Data connection This will run as a $\mathrm{FIFO}^{7}$ based connection. An empty flag is used to identify whether data can be read or not, whilst a read enable will trigger readout of a block of data. Readout should be done from all buffers connected with that single connection, such that data for a single fragment is delivered. Read enable should herein act as a token, being handed from one data-receiver to the next within a block of four, allowing simple readout control and running close to full data rate. The link speed will be $80 \mathrm{Mbit} / \mathrm{s}$ and data line.

S-Link The S-Link interface will be delivered with a HOLA interface, but running data lines with 16 bit width at $40 \mathrm{MHz}$ double data rate. The general interface logic blocks on ROD and BOC side will therefore stay as they are and can easily be adapted to the former system.

\footnotetext{
${ }^{7}$ First In First Out
} 


\section{Testbed}

A testbed for readout system firmware implementation will be set up from two individual evaluation boards (c.f. Figure 3). The right hand side board will emulate ROD functionality, including a PowerPC ${ }^{\circledR}$ processor to develop a first set of firmware to run the initial system. The left hand side board is to implement the BOC functionality checking communication with the detector frontend, as well as with the readout driver. First versions of the IBL readout will thereby soon be tested for operation, needs and misconception.

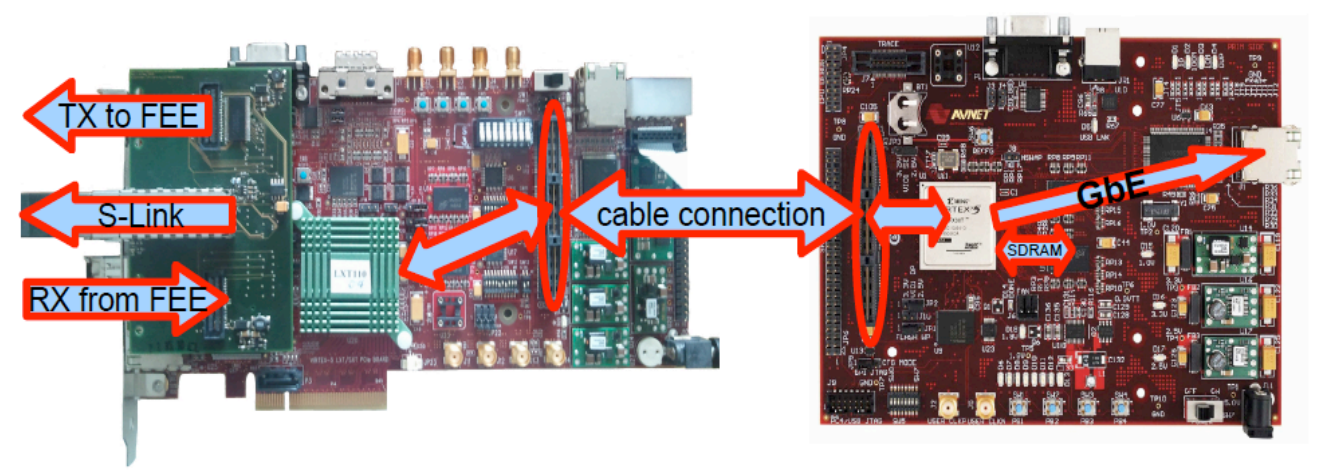

Figure 3. Two different development boards will serve for an initial test setup, delivering a PowerPC ${ }^{\circledR}$ processor on the ROD emulation and large logic space as well as a custom I/O board on the BOC emulation.

\section{Conclusions}

We are proposing a system that delivers the full IBL readout within a single 9U VME crate. Within this paper we described the basic functional design for the I/O interface of the IBL readout system, introducing automated operation of all links, easy exchange of components and a higher bandwidth system.

\section{Acknowledgments}

We like to thank the German Federal Ministry of Education and Research (BMBF) for funding the IBL BOC project as well as the I.N.F.N. for their support. Additionally we have to thank J. M. Joseph for his help in developing ideas and keeping us in contact with the Pixel Detector Subsystem hardware, as well as M. Garcia-Sciveres and M. Barbero for helping us out on FE-I4 topics.

\section{References}

[1] G. Aad et al., ATLAS Pixel Detector Electronics and Sensors, 2008 JINST 3 P07007

[2] The IBL Collaboration, ATLAS Insertable B-Layer Technical Design Report, CERN-LHCC-2010-013

[3] FE-I4 Collaboration, The FE-I4A Integrated Circuit Guide

[4] G. Bruni et al., ATLAS IBL: Integration of new $h w / s w$ readout features for the additional layer of pixels, 2010 JINST proceedings to be published 